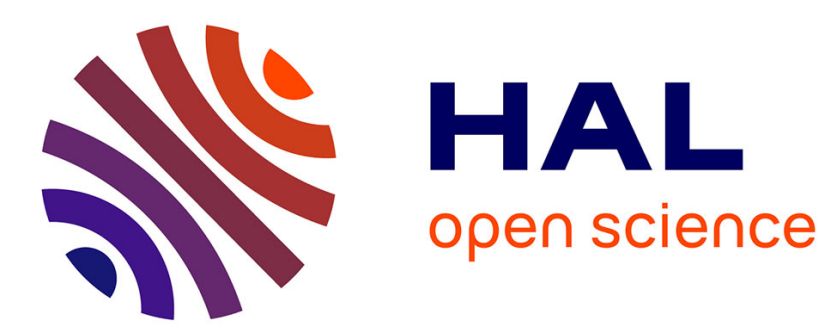

\title{
Analysis of the Chirality Effects on the Capacity of Wireless Communication Systems in the THz band
}

\author{
Anna Maria Vegni, Valeria Loscrì
}

\section{To cite this version:}

Anna Maria Vegni, Valeria Loscrì. Analysis of the Chirality Effects on the Capacity of Wireless Communication Systems in the THz band. IEEE Transactions on Wireless Communications, 2017, 16 (12), pp.7848 - 7858. 10.1109/TWC.2017.2754259 . hal-01589268

\section{HAL Id: hal-01589268 \\ https://hal.science/hal-01589268}

Submitted on 18 Sep 2017

HAL is a multi-disciplinary open access archive for the deposit and dissemination of scientific research documents, whether they are published or not. The documents may come from teaching and research institutions in France or abroad, or from public or private research centers.
L'archive ouverte pluridisciplinaire HAL, est destinée au dépôt et à la diffusion de documents scientifiques de niveau recherche, publiés ou non, émanant des établissements d'enseignement et de recherche français ou étrangers, des laboratoires publics ou privés. 


\title{
Analysis of the Chirality Effects on the Capacity of Wireless Communication Systems in the $\mathrm{THz}$ band
}

\author{
Anna Maria Vegni, Senior Member, IEEE, and Valeria Loscrí, Senior Member, IEEE
}

\begin{abstract}
The potentialities of Terahertz frequency band in the context of nano-scale communications are largely increasing, thanks to specific features that allow to overcome the issues related to the spectrum scarcity and capacity limitation. Apart from high molecular absorption and very high reflection loss that represent the main phenomena in Terahertz (THz) band, in this paper we investigate the chirality effects that affect the propagation medium, in the frequency range $(4-10)$ THz. It is observed that in this interval the chiral parameter shows resonance peaks in specific frequencies.

In this paper we investigate the channel capacity in a special medium affected by chirality effects, such as biomolecules, DNA chains, etc. Specifically, we analyze the signal propagation in a chiral medium where a Giant Optical Activity (GOA) is present. This effect is typical of the so-called chiral-metamaterials. Through simulation results we distinguish the behavior of a chirality-affected channel with GOA in Line-of-Sight and NonLine-of-Sight propagations, assuming different power allocation techniques and also comparing the performance to the case of No GOA.
\end{abstract}

Index Terms-THz band, chiral channel, Giant Optical Activity, nano-communications.

\section{INTRODUCTION}

$\mathbf{N}$ OWADAYS, the growing demand of nanocommunication systems has motivated many researchers to investigate towards the framework of Internet of NanoThings [1], with the increasing need to design accurate channel models working in the Terahertz band [2]. The Terahertz band has been identified in the range $(0.06-10) \mathrm{THz}[3]$, and represents one of the most promising spectrum bands to enable ultra-high-speed communications. Indeed, to overcome the spectrum scarcity and capacity limitations of current wireless systems, many researchers are investigating the potentialities of the $\mathrm{THz}$ band. Also, since existing channel models adopted for lower frequency bands are unsuitable for $\mathrm{THz}$ communications, the need of novel channel models specific for this frequency range is a challenge.

Recently, several studies have investigated the channel behavior in the $\mathrm{THz}$ band [4], [5], [6], [7], [8], [9], [10], [11]. Specific features are evident when working in optic and almost-optic frequencies, like the very high molecular absorption loss or the very high reflection loss. Differently from traditional lower frequency bands, where the propagation is mainly influenced by the spreading loss only, the physical

A. M. Vegni (corresponding author) is with the Department of Engineering, Roma Tre University, Rome, Italy, e-mail: annamaria.vegni@uniroma3.it

V. Loscrí is with INRIA, Lille-Nord Europe, Lille, France. e-mail: valeria.loscri@inria.fr

A much shorter version of this work appears in the Proc. of ACM NANOCOM 2016, New York City, New York, USA, September 28-30, 2016. mechanisms in a $\mathrm{THz}$ band wireless transmission are $(i)$ a very high molecular absorption loss and (ii) the spreading loss. The first concept has been broadly surveyed in [12], where the authors distinguish the time and frequency dependence. Jornet and Akyilidz in [4] present the effect of molecular absorption on the total system noise, by assuming different types and concentrations of molecules. The authors also show the effect of molecular absorption on the path loss and the channel capacity. In [5], Javed and Naqvi introduce SimpleNano that is an approximation to log-distance path loss model along with the random attenuation caused by the molecular absorption. The authors show that for certain band windows within the $\mathrm{THz}$ range SimpleNano experiences low molecular noise. Finally, for short-range $\mathrm{THz}$ communications Llatser et al. [6] study the influence of molecular absorption on both frequency and time domain.

Several works have investigated $\mathrm{THz}$ band channel in indoor environments, such as [13], [14]. In [15] the authors investigate the channel behavior in $\mathrm{THz}$ band for indoor environments. Similarly, Khalid and Akan [16] present experimental results of a $2 \times 2$ multiple-input multiple-output channel in $\mathrm{THz}$ band line-of-sight propagation. However, $\mathrm{THz}$ band can find interesting applications not only in indoor environments. For instance, the concept of spreading loss is exploited to derive the path loss behavior in the THz band, like in [7] where Yang et al. present the performance of an electro-magnetic (EM) channel for applications in body area networks. Other works present channel models for specific applications and contexts, like nanomedicine in [8] and graphene-based systems [9].

Furthermore, apart the study of specific features of channel model in $\mathrm{THz}$ band, it is also interesting to observe how the signals propagate into the medium, under the hypothesis of Line-of-Sight (LoS) and Non-Line-of-Sight (NLoS) propagation. Indeed, a very high and frequency selective path loss are experienced in LoS links, while high reflections occur in NLoS propagation due to the type of material and the roughness factor of reflecting surfaces [15], [17], [18], [10]. For instance, Han et al. [18] consider a multi-ray approach by assuming reflected, scattered and diffracted paths, in order to model the main $\mathrm{THz}$ band channel features, such as the distance-varying spectral windows and the temporal broadening effects.

Leveraging on all previous related works, we notice that the channel modeling in $\mathrm{THz}$ band is mainly addressed through molecular absorption loss and spreading loss. However, other effects deserve to be considered and analyzed, such as the electromagnetic chirality effect and the specific features of the propagation medium, that are normally neglected in classical modeling of transmission channels. The relative chirality pa- 
rameter is typical of a chiral homogeneous isotropic medium i.e., a medium with chiral impurities homogeneously and spatially distributed inside the host material [19]. Standard values are positive and lower than or equal to 1 , but in particular media such as the chiral complex materials the relative chirality parameter becomes complex, with a frequency-dependent resonant behavior [20]. In this particular type of material, it is observed an enhanced rotation of the light polarization plane, known as Giant Optical Activity (GOA) [21]. A material exhibiting a GOA and affected by the chirality effects is called chiral metamaterial [22], i.e., an artificial material that shows a frequency-dependent cross-coupling between the electric and magnetic fields. The study of performances of a GOA-affected material may be important for the propagation of the $\mathrm{THz}$ signals through stratified chiral-metamaterials.

We remind that metamaterials are unconventional materials, that is, artificial structures able to generate unique physical phenomenon such as the negative refraction index and backward waves [23], [24]. Typically, the properties of metamaterials have been utilized in the implementation of invisible cloaks, for sub-wavelength imaging [25], wireless power transfer [26], and antenna miniaturization [27]. In the context of wireless communications, the use of metamaterials could find effective applications [28], [29]. In [30], the authors present the channel characteristics (i.e., channel capacity and path loss) of a metamaterial-enhanced magnetic induction communication model, in case of a point-to-point and waveguide communication system. The proposed system applies to various environments that are structurally complex and challenging for RF wireless signals.

As next step, the use of chiral metamaterials for wireless communications in $\mathrm{THz}$ band is worth to be investigated. Chiral metamaterials represent perfect candidates for nanosystem applications realization, and more specifically, for all those operations in the $\mathrm{THz}$ and also higher frequencies. In fact, in comparison to natural materials, chiral metamaterials show strong response to the $\mathrm{THz}$ radiation, thus providing a great technological potential in several sectors such as imaging, sensing and also communications, by accordingly designing and fabricating a metamaterial structure [31], [32], [33]. Also, materials exhibiting a GOA show a huge potential in the achievement of active $\mathrm{THz}$ polarization components [34]. GOA consists in the ability to rotate the polarization state of light. This effect is typical of chiral structures such as DNA, bio-molecules, sugar solutions, etc. Thanks to the recent technological advancements, the possibility to control light polarization allows the realization of metamaterials that show a much stronger chirality than in the natural media. As a result, the corresponding devices can be ultrathin and miniaturized, suitable for lab-on-chip integration.

Finally, about fabrication of chiral metamaterials, generally, they are fabricated according to (i) the top-down and (ii) bottom-up approaches. In the top-down approach, various techniques are used, such as ion beam lithography, electron beam lithography and direct laser writing, in order to accordingly cut, mill and shape materials. On the other side, the bottom-up approach usually arranges small components into more complex assemblies. The interested reader can find more

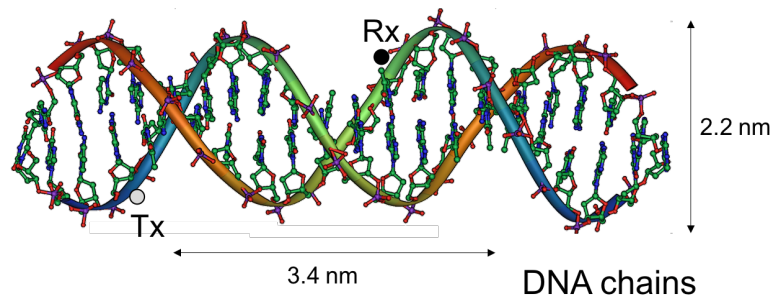

(a)

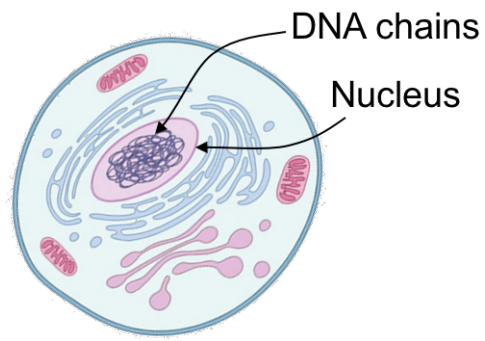

(b)

Fig. 1. Schematics of a biological environment for nanoscale communications, where the chirality effect can be found. (a) Structure of DNA chains with a transmitter and a receiver connected to each others. (b) In-cell communications in an eukaryotic cell.

information in [35], [36], [37].

In this paper, we investigate how the chirality effects affect the channel capacity of a metamaterial, in order to pave the realization of chiral-based devices to fill the $\mathrm{THz}$ gap. For the best of our knowledge, chiral metamaterials have not been investigated yet in the context of $\mathrm{THz}$ band communications, and therefore represent a novel research topic. Among the main domains suitable for the use of chiral metamaterials, biomedicine is one of the most likely, i.e., preventive healthcare, surgery, quality control, nondestructive evaluation, tumor diagnostic, etc. Indeed, the electromagnetic propagation of $\mathrm{THz}$ signals through metamaterials fits well in biological environments (e.g. deep implanted antennas in living matter or DNA channel modeling). An example of the biological context where the chirality effect can be found is depicted in Fig. $1(a)$, with a transmitter and a receiver connecting to each other. In such a scenario, data can be transmitted along DNA chains, which can be modeled as a chiral medium (i.e., electric dipoles interconnected with magnetic loops) due to their specific double helix shape [38].

From the above considerations, we envisage the use of chiral metamaterials as a propagation medium along DNA strands, and -more in general- for in-cell communications. Indeed, each living cell contains DNA strands. For instance, in an eukaryotic cell -as depicted in Fig. $1(b)-$, DNA is enclosed in a membrane, which forms a structure called the nucleus.

It is worth to clarify that the use of chiral metamaterials as propagation medium does not represent an alternative to freespace propagation. Our purpose is to study the features of chiral metamaterials since several propagation environments (e.g., the human body) can be modeled by assuming the presence of the chiral effect. For example, in order to model the DNA helix chains as propagation medium we can use a 
cascade of equivalent transmission lines, each one loaded with omega loops that are acting as localized magnetic dipoles. In this case the propagation medium is, clearly, not the freespace and the relative equivalent model is a meta-structure (that is a metamaterial propagation channel). Therefore, a more appropriate channel model based on chiral metamaterials is necessary. A deeper comprehension of the $\mathrm{THz}$ signals in chiral metamaterial can pave the realization of effective artificial bio-molecules to be exploited as information carrier in several domain spanning from medicine, agriculture, etc.

Recently, in [39] we investigated the chirality effects occurring in a chiral metamaterial, and introduced them into a full-wave propagation model of the electromagnetic field that propagates in the $\mathrm{THz}$ band. Then, we derived how the channel transfer function is affected by such chiral effects. Results about path loss in LoS and NLoS propagation modes, in case of biological and indoor application scenarios, have been presented in order to validate the proposed study. Also, in [40], we analyzed the specific behavior of transfer functions in a chiral-metamaterial channel exhibiting a GOA, in case of direct and multi-path propagation. Performance results were assessed in terms of capacity, propagation delays and coherence bandwidths.

All the previous results pushed us to keep on investigating on this unconventional material for communications in the $\mathrm{THz}$ band. Specifically, we observed that the tunability of a chiral matematerial allows to maximize the network performances for specific frequency sub-bands.

In this paper, we refer to our previous works [39], [40], [41] as a starting point to develop a channel model for nanocommunication applications in the $(4-10) \mathrm{THz}$ frequency band, when an artificial (metamaterial) propagation medium is present. Specifically, in [39] we introduced the chirality effects in the full wave model of $\mathrm{THz}$ signal propagation, and derived how the channel transfer function is affected by this particular effect. Results have been presented in terms of path loss in different scenarios and propagation modes. The next work [40] addressed the derivation of the other performances of a chiral metamaterial, i.e., the channel capacity, the propagation delay, and the coherence bandwidth.

Now, in this paper, we investigate how the channel of a chiral metamaterial responds to different inputs (i.e., pulse waveforms), thus achieving the channel capacity bounds in case of different power allocation schemes. As outlined in [40], due to the resonant behavior of the chiral parameter, the channel model in a chiral metamaterial shows specific frequencydependent spectral windows, guaranteeing very high bandwidth values up to $6 \mathrm{THz}$ that represents the maximum amount of bandwidth in the range $(4-10) \mathrm{THz}$. In such a material, it is of very useful to investigate the capacity behavior. The main contributions of this paper are enumerated as follows:

1) To deeply motivate the reader about the choice of such an unconventional channel for nanocommunication purpose. Indeed, chiral metamaterials can be applied for nanoscale communications. They do not represent an alternative propagation medium to free-space, instead they need to be considered in specific scenarios where the chiral effects occur (e.g., when a biological propagation medium needs to be studied). This aspect is not present in previous works [39], [40];

2) To develop a channel model for EM nanocommunications in a chiral metamaterial in the frequency range $(4-10) \mathrm{THz}$;

3) To propose different power allocation patterns that are useful for evaluating the performance of the chiral metamaterial, expressed in terms of channel capacity;

4) To present extensive simulation results by comparing the channel capacity bounds under different power spectral densities and for LoS/NLoS propagation modes. Also, a comparison of performances to the case of No GOA is shown. This aspect was not considered in [39], and only marginally in [40].

This paper is organized as follows. In Section II we briefly present the frequency-dependent behavior of specific parameters of a chiral-metamaterial in the $\mathrm{THz}$ band. Indeed, the chiral impurities inside the propagation medium cause a change in the propagation velocity and in the refractive index, which reflect in a specific behavior of the channel transfer function. The chirality effects are evaluated also in the case when the considered medium exhibits a GOA [20], [42]. In Section III we derive the corresponding chirality-affected channel model for $\mathrm{THz}$ band, and present the frequencydependent path loss in a chiral medium both with and without GOA. Section IV is then devoted to the performances of the chiral channel, assessed in terms of capacity per subband for different power allocation schemes and distances, in case of LoS and NLoS propagation modes, with and without GOA. We evince that best performances are obtained for LoS propagation in a medium exhibiting GOA. Finally, conclusions are drawn at the end of the paper.

\section{Full-WaVe Propagation in A CHIRAL-METAMATERIAL}

The design of a full-wave propagation model for $\mathrm{THz}$ band within a medium affected by GOA is a complex task that requires information about the main characteristics of the propagation channel.

Unconventional materials (i.e., metamaterials) are special materials where the GOA is reinforced by using thin metallic crossed-structure impurities in the host dielectric medium. These specific materials are known as chiralmetamaterials [22]. A chiral metamaterial shows that the displacement field existing inside the material is generated by an excitation expressed in terms of intensity of the incident electro-magnetic field. It follows that the chiral effects have a two-fold meaning, i.e., ( $i)$ an electric field applied on the material provides not only an electric induction, but also a magnetic displacement, and (ii) a magnetic field applied on the material provides not only a magnetic induction, but also an electric displacement, unlikely from other non-chiral materials.

Chirality effects are shown in composite materials where the same handed wire-loop inclusions are distributed uniformly and randomly. This means that the material constitutive relations are changed with the addition of a third characteristic parameter (i.e., the chirality parameter) besides the dielectric permittivity and the magnetic permeability. The main 
effect of chirality occurring in the $\mathrm{THz}$ frequency band e.g., $(0.06-10) \mathrm{THz}$, is the explosion of chiral parameter that reaches values up to 40 times the normal ones, giving rise to GOA.

Starting from the classic harmonic macroscopic Maxwell's equations. we can consider the electromagnetic propagation inside a generic complex material, under the assumption that it is a linear and chiral medium. In such a material, chiral (magneto/electric-optical) effects occur according to the following constitutive relations:

$$
\left\{\begin{array}{l}
\mathbf{B}=\underline{\xi} \bullet \mathbf{E}+\mu \bullet \mathbf{H} \\
\mathbf{D}=\underline{\varepsilon} \bullet \mathbf{E}+\underline{\zeta} \bullet \mathbf{H}
\end{array}\right.
$$

where $\mathbf{B}$ and $\mathbf{H}$ are the magnetic displacement and the magnetic field, respectively, while $\mathbf{D}$ and $\mathbf{E}$ are the electric displacement and electric field, respectively. Also, the symbol - represents the scalar product operator and $\underline{\xi}, \underline{\mu}, \underline{\varepsilon}$, and $\underline{\zeta}$ are specific tensor quantities of the material. From (1) we observe the chirality property through the dependence of $(i) \mathbf{E}$ in $\mathbf{B}$, and (ii) $\mathbf{H}$ in $\mathbf{D}$.

For a GOA reciprocal material the specific constitutive relations are as follows [43], [44]:

$$
\left[\begin{array}{l}
\mathbf{B} \\
\mathbf{D}
\end{array}\right]=\left[\begin{array}{cc}
-j \frac{\xi_{0} \xi_{r}}{c} & \mu_{0} \mu_{r} \\
\varepsilon_{0} \varepsilon_{r} & j \frac{\xi_{0} \xi_{r}}{c}
\end{array}\right] \bullet\left[\begin{array}{c}
\mathbf{E} \\
\mathbf{H}
\end{array}\right],
$$

where it is clear that the natural dielectric (where $\varepsilon_{r}$ and $\mu_{r}$ are the relative electrical permittivity and magnetic permeability, respectively) becomes a metamaterial with $\varepsilon_{r}$ and $\mu_{r}$ the relative permittivity and permeability, respectively, while $c$ is the light speed, $\varepsilon_{0}$ and $\mu_{0}$ are the vacuum permittivity and permeability, respectively, and $\xi_{r}$ is the relative chirality parameter. Moreover, for a GOA material with specific features and impurities as depicted in [20], at the frequency around $5 \mathrm{THz}$ and $8 \mathrm{THz}$, there are four resonance frequencies of the $\xi_{r}$ relative chirality parameter, i.e., $[4.8,5.6,7.9,8.2] \mathrm{THz}$. A similar consideration is applied to the relative permittivity and permeability parameters, still in the $(4-10) \mathrm{THz}$ band. As reported in [20], $\varepsilon_{r}$ and $\mu_{r}$ are complex parameters, whose real parts have a frequency-dependent behavior with resonant peaks.

Notice that the proposed structure in [20] was based on a stack of a planar dielectric (natural) slabs doped via metallic impurities. The resulting structure is a metamaterial one, with untraditional electromagnetic properties. It has been proposed for novel terahertz device applications in the $\mathrm{THz}$ frequency range, such as wave plate, ultrathin polarizer, band pass filters, and others.

Finally, for sake of simplicity we assume that the GOA channel is excited by a linear polarized electric field, whose definition is obtained by inserting the specific GOA constitutive relations (2) in the Maxwell's equations and solving the relative partial differential equation, i.e.,

$$
\left\{\begin{array}{l}
{\left[(\underline{\nabla}-j \omega \underline{\zeta}) \bullet \underline{\mu}^{-1} \bullet(\underline{\nabla}+j \omega \underline{\xi})-\omega^{2} \underline{\varepsilon}\right] \bullet \mathbf{E}=0} \\
{\left[(\underline{\nabla}+j \omega \underline{\xi}) \bullet \underline{\varepsilon}^{-1} \bullet(\underline{\nabla}-j \omega \underline{\zeta})-\omega^{2} \underline{\mu}\right] \bullet \mathbf{H}=0}
\end{array}\right.
$$

where $\underline{\nabla}$ is the well-known Kong's vector.

Notice that the proposed EM model is a like-Drude one and shows only one resonance frequency [45]. In the Drude model, the effective electric permittivity $\varepsilon_{r}$ acts according to a theoretical model, well confirmed by the experiments, where one or more resonance frequencies appear [29].

However, since for a GOA medium in the frequency range $(4-10) \mathrm{THz}$ we observe four resonances for chiral parameter, as well as for the electric permittivity and magnetic permeability, as depicted in [39], it follows that the proposed EM model can be easily extended to a more complex one, like a Drude-Lorentz [45].

\section{Chiral Channel Model with GOA}

After describing the main features of a chiral metamaterial from the electro-magnetic propagation point of view, we now investigate how the relative chiral parameter $\xi_{r}$ affects the channel performance in the $(4-10) \mathrm{THz}$ band, under the hypothesis of ray tracing propagation. Indeed, in Section II we firstly derived the Drude-like EM model for a GOA channel; then, we keep on exploiting Maxwell's equations to obtain the chirality dependence of the channel transfer functions in LoS and NLoS scenarios. Specifically, in NLoS case, we focus on reflected paths only due at generic reflection centers located at $z$-plane, while omitting scattered, and diffracted rays. The reflection characteristics of the transmissive channel can be evaluated through the specific knowledge of the local planar geometry associated to the reflection centers.

Following the multi-ray model in [10], let us model the channel as the combination of several individual narrow subbands, each of them with a flat-band response. In the $i$-th frequency sub-band, the channel impulse response is expressed as a superposition of $N$ rays. In the case of stationary environment, the channel response in the $i$-th sub-band is given as:

$$
h_{i}(\tau)=\sum_{n=1}^{N} \alpha_{i, n} \delta\left(\tau-\tau_{n}\right),
$$

where $\alpha_{i, n}$ is the frequency-dependent attenuation of the $n$-th ray, and $\tau_{n}$ is the propagation delay of the $n$-th ray in NLoS mode.

Following the well-known schemes of LoS and NLoS propagation modes, from (4) we can derive the LoS and NLoS channel transfer functions in the case of a chirality-affected channel with GOA, respectively as [39]:

$$
H_{\mathrm{LoS}}(f)=H_{\mathrm{Abs}}(f) H_{\mathrm{Spr}}(f) e^{-j 2 \pi f \tau_{\mathrm{LoS}}} \text {, }
$$

and

$H_{\mathrm{NLoS}}(f)=\left[\frac{\nu_{c}}{4 \pi f\left(d_{1}+d_{2}\right)}\right] e^{-j 2 \pi f \tau_{\mathrm{NLoS}}-\frac{1}{2} k(f)\left(d_{1}+d_{2}\right)} \cdot R(f)$,

where we assume the NLoS scenario is affected by reflected rays only through the rough surface reflection loss, i.e., $R(f)$, which depends on the material, the shape and the roughness of the surface on which the EM field has been reflected. Specifically, in the simulation results we considered the reflection coefficient for the plaster as a function of the incident angle, as reported in [10]. To give an example, a constant value of $-23 \mathrm{~dB}$ has been assumed for an incident angle of $22^{\circ}$. Information about the related experimental setup can be 
found in [10], where several examples of validation of multiray model with experimental measurements are shown. Also, in [46], a general measurement system is presented and a large amount of experimental data are shown.

We can notice that in the previous equations (5) and (6) the chirality factor appears through the propagation velocity of the electromagnetic field in a chiral homogeneous isotropic medium, i.e., $\nu_{c}$. Indeed, in (5), $H_{\mathrm{Abs}}$ represents the transfer function due to the molecular absorption loss, while $H_{\mathrm{Spr}}$ is the transfer function due to the spreading loss that takes account for the chirality effect as follows:

$$
H_{\mathrm{Spr}}=\frac{\nu_{c}}{4 \pi f r},
$$

where $r$ is the distance in LoS between the transmitter and the receiver. The expression of $\nu_{c}$ can be easily obtained as

$$
\nu_{c}=\frac{c}{n_{c}}
$$

where $c$ is the light propagation speed and $n_{c}$ is the refractive index in a chiral medium, defined as:

$$
n_{c}=\sqrt{\mu_{r} \varepsilon_{r}+\xi_{r}^{2}},
$$

with $\mu_{r}, \varepsilon_{r}$ and $\xi_{r}$ frequency-dependent parameters [40], [41]. The demonstration of previous result is provided in a dedicated Appendix in our recent work [39]. It follows that $n_{c}$ is frequency-dependent. Notice that the electrical permittivity and the magnetic permeability are generally frequencydependent for dispersive materials. The chirality factor $\xi_{r}$ is generally frequency-independent unless the used material presents a GOA as it happens in chiral metamaterials (see e.g., [47], [48], [49]).

From the expressions of channel transfer functions in (5) and (6), we can compute the total path loss, as depicted in Fig. 2 (a) and (b) in the case of LoS and NLoS propagations, respectively. We assume different distances from transmitter to receiver, and a specific reflecting angle for multi-path scenario (i.e., $22^{\circ}$ ). We also compare the path loss in case of (i) GOA, and (ii) No GOA scenario. For the case of No GOA, we assumed $\xi_{r}=1, \varepsilon_{r}=1$, and $\mu_{r}=4$.

As known, the total path loss of a propagating wave in the $\mathrm{THz}$ band depends on different parameters, such as the frequency and the distance, as well as the composition of the propagating medium at a molecular level. However, in Fig. 2, we omit the frequency-dependent behavior of molecular absorption, in order to better highlight the chirality effects that affect the path loss in specific resonance frequencies. We notice that for increasing distances, the path loss has a higher trend. Furthermore, similarly to the results in [18] and independently from GOA, the LoS propagation in Fig. 2 (a) provides lower values of path loss with respect to the NLoS scenario depicted in Fig. 2 (b). In both LoS and NLoS, the behavior is frequency-dependent, but in LoS propagation the path loss has a smoother trend, with some low peaks at 5.8, 8.09 and $8.93 \mathrm{THz}$. On the other hand, for NLoS propagation, higher values are well noticeable at 5.8 and $8.93 \mathrm{THz}$, while on the other frequencies, the trend is on average flat around 35,40 and $45 \mathrm{~dB}$ for $d=1,5$, and $10 \mathrm{~mm}$, respectively. The curves for No GOA show a slow increasing behavior of path

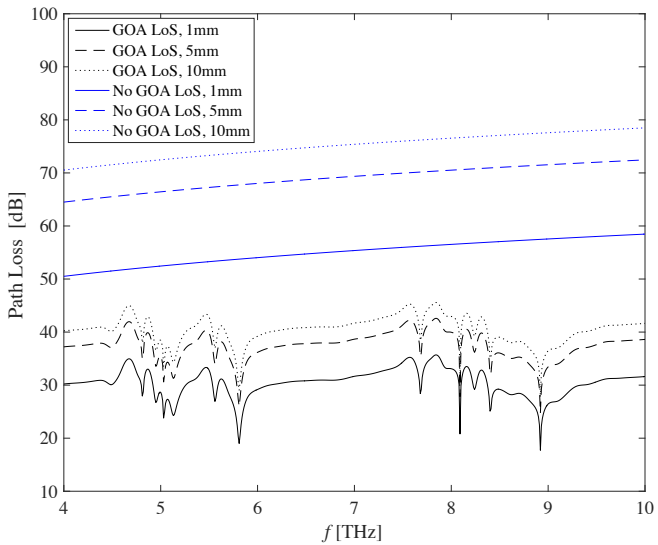

(a)

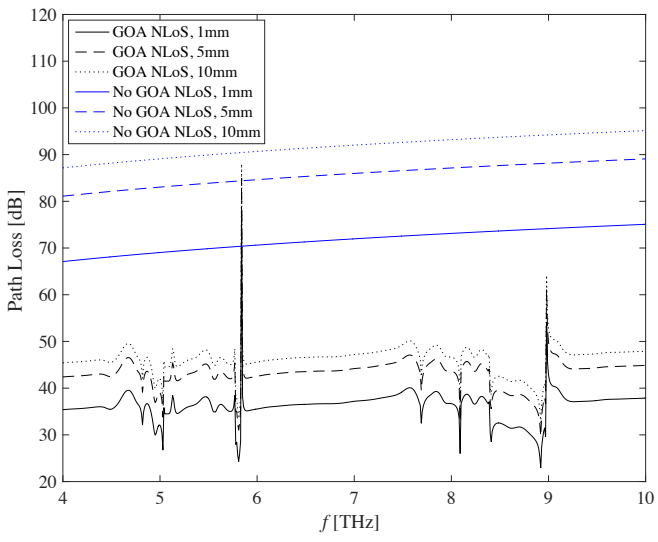

(b)

Fig. 2. Path loss in a chiral channel in case of (a) LoS, and (b) NLoS propagation, for GOA and No GOA (i.e., $\varepsilon_{r}=1, \mu_{r}=4$, and $\xi_{r}=1$ ) scenarios, and different distances.

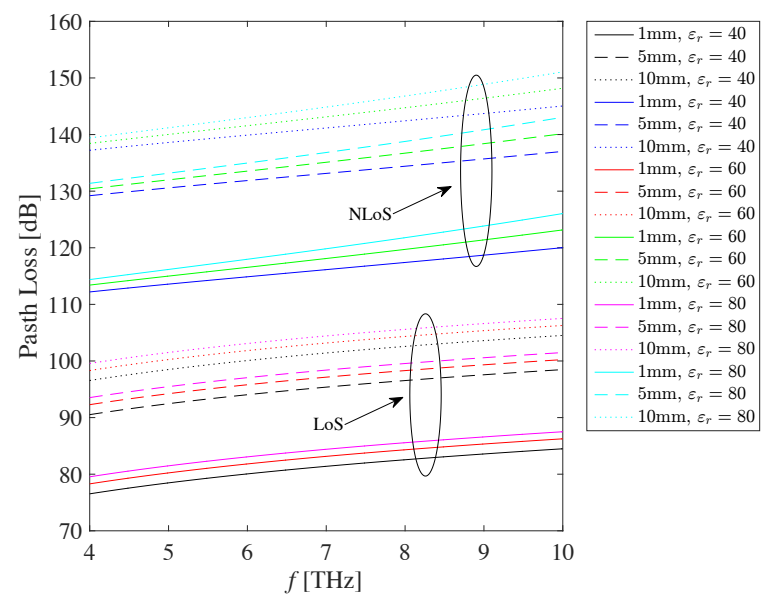

Fig. 3. Path loss vs. frequency in a medium without chiral effect, for different values of $\varepsilon_{r}$ and distance, in case of LoS and NLoS.

loss versus the frequency. We also observe higher values of path loss in case of No GOA (blue lines) with respect to GOA curves (black lines), except for a few peaks of path loss, as depicted in Fig. 2 (b) around 5.8 THz. This is mainly due to the frequency-dependent behavior of channel transfer functions in case of GOA. 
Finally, in order to highlight how the chiral effect influences the path loss, in Fig. 3 we show the behavior of a metamaterial channel without chiral effects in case of LoS and NLoS propagation and different distances. As expected, the NLoS case has lower performances, which gets worse for increasing distances. However, the absence of chiral effect provides a flatter behavior of the path loss, approximable to the case of $\xi_{r}=1$ in Fig. 2 . In contrast, the GOA provides a very variable behavior of path loss w.r.t. frequency.

\section{Capacity Analysis of a Chiral Channel}

Following the chirality-affected channel model presented in Section III, we now investigate the capacity behavior in the (410) $\mathrm{THz}$ band. Specifically, we focus on the channel capacity in case of LoS and NLoS propagation and under different assumptions for power spectral density (p.s.d.) within the whole band of interest. Since in this paper our aim is basically addressed to the channel capacity behavior in LoS and NLoS propagation in a chiral-affected medium, in the simulation results, we assume a flat behavior for the molecular absorption loss, i.e., frequency-independent behavior [12]. Thus, the following results represent the behavior of channel capacity per sub-band along LoS and NLoS directions in a chiralityaffected channel, by omitting the frequency-dependent molecular absorption effect. Results have been obtained in Matlab ${ }^{\circledR}$ environment.

The capacity limits in a chiral medium can be assessed by assuming the received signal as a sum of each contribution in the $i$-th sub-band, each one with a narrow behavior and a flat-band response, so that the following constraint is adopted:

$$
\sum_{i=1}^{N_{B}} P_{i} \leq P_{T O T}
$$

where $N_{B}$ is the total number of sub-bands, $P_{i}$ is the transmission power in the $i$-th sub-band, and $P_{T O T}$ is the total transmit power in the $(4-10) \mathrm{THz}$ band. Notice that, since the chiral parameter has a frequency-dependent behavior in $(4-10) \mathrm{THz}$ band, we consider only this frequency range. Then, the overall capacity $C$ can be defined as the sum of the single capacities in each of $N_{B}$ sub-bands, i.e.,

$$
C=\sum_{i=1}^{N_{B}} C_{i}=\sum_{i=1}^{N_{B}} \Delta f_{i} \log \left(1+\frac{\left|h_{i}\right|^{2} P_{i}}{\Delta f_{i} S_{N}\left(f_{i}\right)}\right),
$$

where $S_{N}$ is the power spectral density of the additive white Gaussian noise and $\Delta f_{i}$ is the sub-band range among two consecutive sub-bands, i.e., $\Delta f_{i}=f_{i+1}-f_{i}$. Specifically, in the whole range $(4-10) \mathrm{THz}$ we assume a gap of $10 \mathrm{GHz}$ among two consecutive sub-bands and we obtain $N_{B}=600$. We remark that in this paper our aim is to investigate the channel capacity in a chiral metamaterial when a GOA is present. This is accomplished through the derivation of the channel capacity in (11), which is affected by the chiral effect and the transmission power level. Also, the power profile in the $i$-th sub-band has a frequency-dependent behavior, and we can accordingly tune the working frequency in order to enhance the channel performances. More in detail, since the channel capacity per sub-band in (11) strictly depends on

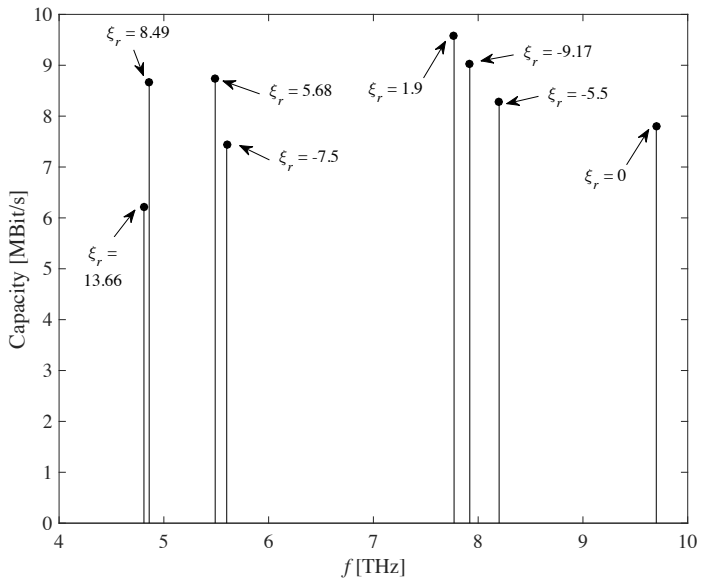

Fig. 4. Channel capacity per sub-band for different parameters of chiral metamaterials. Table I collects the values of $\varepsilon_{r}$ and $\mu_{r}$ corresponding to the obtained results.

TABLE I

LIST OF PARAMETERS USED IN FIG. 4.

\begin{tabular}{|c|c|c|c|}
\hline$f[\mathrm{THz}]$ & $\varepsilon_{r}$ & $\mu_{r}$ & $\xi_{r}$ \\
\hline 4.81 & -54.27 & 3.26 & 13.66 \\
\hline 4.86 & -38.46 & 3.65 & 8.49 \\
\hline 5.49 & 21.50 & 2.18 & 5.68 \\
\hline 5.6 & -15 & 2.18 & -7.5 \\
\hline 7.77 & -18.82 & 3.35 & 1.9 \\
\hline 7.92 & -24.81 & 4.83 & -9.17 \\
\hline 8.2 & 2.99 & -2.2 & -5.49 \\
\hline 9.7 & 5.65 & 1.92 & 0 \\
\hline \multicolumn{4}{|c}{} \\
\hline
\end{tabular}

the channel transfer function, i.e., $h_{i}$, as well as the power profile, i.e., $P_{i}$, of the $i$-th sub-band, we expect to observe ( $\left.i\right)$ a frequency-dependent behavior due to the chiral parameter affecting $h_{i}$, and (ii) different values of channel capacity per sub-band depending on a given power allocation scheme adopted in the $i$-th sub-band.

Fig. 4 depicts the channel capacity per sub-band as a function of the relative chirality parameter $\xi_{r}$, the electric permittivity $\varepsilon_{r}$, and the magnetic permeability $\mu_{r}$, each of them occurring at a given frequency, according to Table I. In Fig. 4 we choose different values of $\xi_{r}$, in order to better understand the behavior of the channel capacity per subband. Particularly, we consider the maximum and minimum values of $\xi_{r}$, i.e., $\xi_{r}=13.66$, and $\xi_{r}=-9.17$ occurring at $4.8 \mathrm{THz}$ and $7.9 \mathrm{THz}$, respectively. In addition, we consider the resonance peaks occurring at $5.6 \mathrm{THz}$ and $8.2 \mathrm{THz}$, corresponding to $\xi_{r}=-7.5$ and $\xi_{r}=-5.5$, respectively. Finally, we compute the samples of channel capacity per subband for $\xi_{r}=0, \xi_{r}=5.68, \xi_{r}=8.49$ occurring at 9.7, 5.68 , and $4.86 \mathrm{THz}$. We notice that the channel capacity per sub-band has no linear dependence from the chiral parameter. Indeed, for a given frequency in the range $(4-10) \mathrm{THz}$, there exist different values for $\varepsilon_{r}, \mu_{r}$, and $\xi_{r}$.

In the following, we present the analysis of channel capacity in a chiral metamaterial, assuming different power allocation schemes, in case of GOA and No GOA scenarios. In the expression of channel capacity (11), we can distinguish differ- 


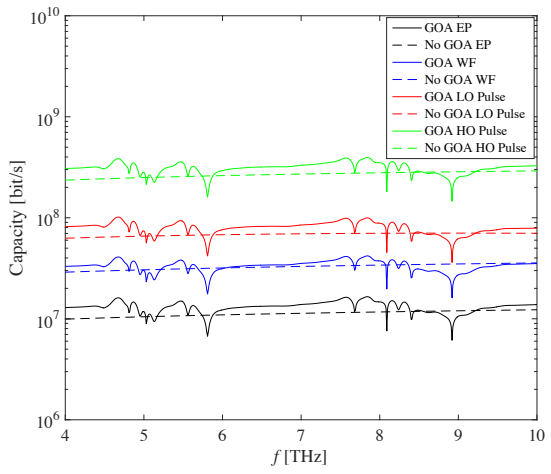

(a)

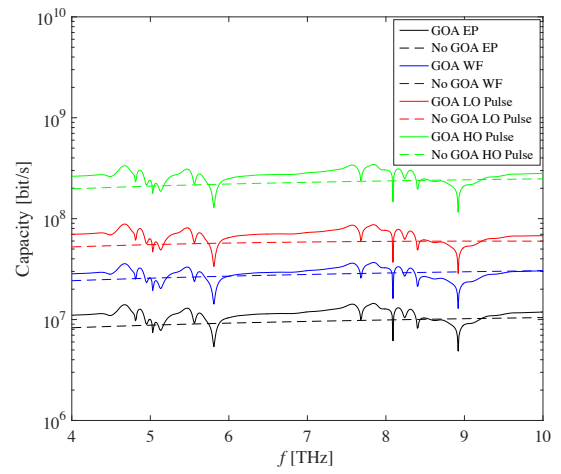

(b)

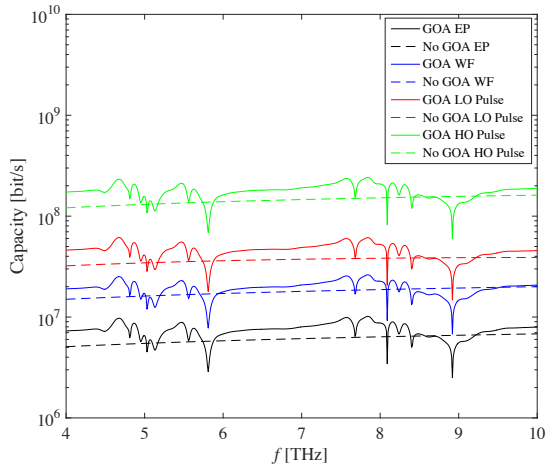

(c)

Fig. 5. Comparison of capacity per sub-band in LoS propagation in the range $(4-10)$ THz, in case of GOA and No GOA assumption, different p.s.d. and at a distance of (a) 1 , (b) 5 , and (c) $10 \mathrm{~mm}$.

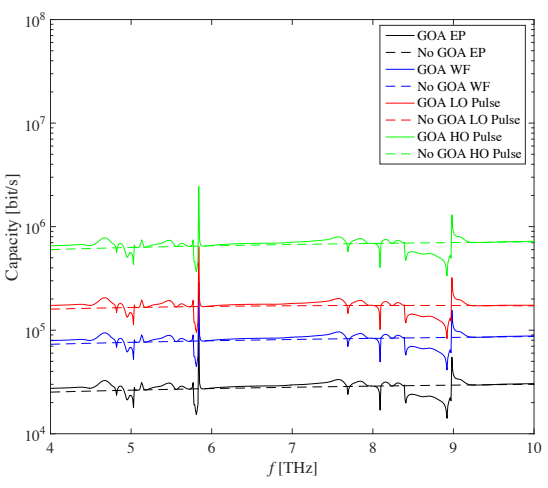

(a)

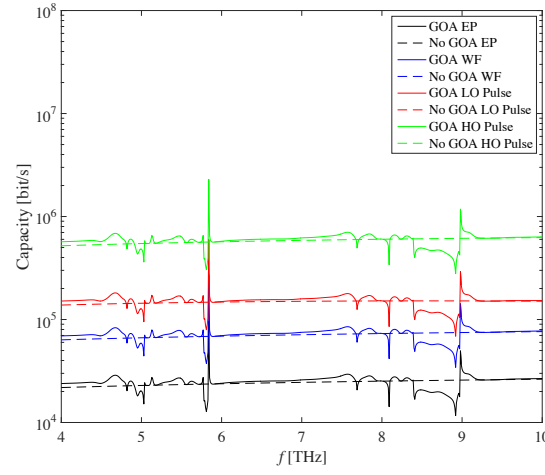

(b)

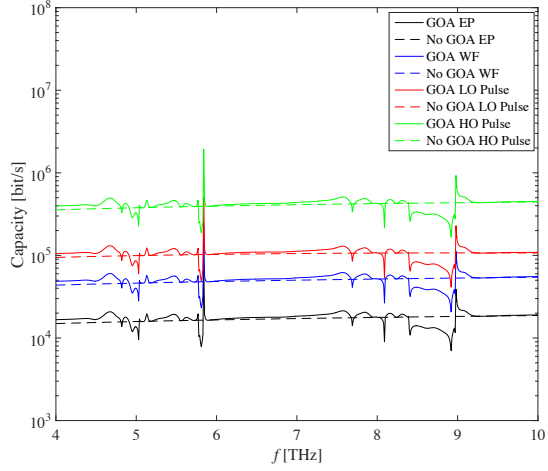

(c)

Fig. 6. Comparison of capacity per sub-band in NLoS propagation in the range $(4-10) \mathrm{THz}$, in case of GOA and No GOA assumption, different p.s.d. and at a distance of (a) 1 , (b) 5 , and (c) $10 \mathrm{~mm}$.

TABLE II

LIST OF MAIN PARAMETERS USED IN THE SIMULATION RESULTS FOR DIFFERENT SCENARIOS AND PROPAGATION MODES.

\begin{tabular}{|lll|}
\hline & LoS & NLoS \\
\hline GOA & Direct connection (1 ray) & Multi-ray model (5 rays) \\
& Frequency dependent be- & Frequency dependent behav- \\
& havior for $\xi_{r}, \varepsilon_{r}$ and $\mu_{r}$ & ior for $\xi_{r}, \varepsilon_{r}$ and $\mu_{r}$ \\
\hline No GOA & Direct connection $(1$ ray) & Multi-ray model (5 rays) \\
& $\xi_{r}=0, \varepsilon_{r}=1, \mu_{r}=4$ & $\xi_{r}=0, \varepsilon_{r}=1, \mu_{r}=4$ \\
\hline
\end{tabular}

ent scenarios according to how the power level is distributed within the whole frequency band. As an instance, in the simplest case, we can assume a flat behavior of power spectral density in the $i$-th sub-band, i.e.,

$$
P_{i}=P_{\text {flat }}(f)= \begin{cases}P_{0}, & \forall f \in B \\ 0, & \text { if } f \notin B\end{cases}
$$

that is, the power transmission is uniformly distributed over the entire operative band (i.e., from 4 to $10 \mathrm{THz}$ ). Specifically, we consider a power level $P_{0}$ equal to $46 \mathrm{dBm}$, divided across all the sub-bands, i.e., $N_{B}=600$. This represents a power allocation scheme where the power level is equally distributed over all the sub-bands -namely, equal power (EP) allocation scheme-.

The EP scheme represents a very simple case that can be improved by accordingly choosing the power level $P_{i}$ in the $i$-th sub-band, following the waterfilling principle (WF), i.e.,

$$
P_{i}=P_{W F}(f)= \begin{cases}\frac{K-\Delta f_{i} \cdot S_{N}(f)}{\left|h_{i}\right|^{2}}, & \text { if } K \geq \Delta f_{i} \cdot S_{N}(f) \\ 0, & \text { if } K<\Delta f_{i} \cdot S_{N}(f)\end{cases}
$$

where $K$ is a constant accordingly chosen based on the total transmitted power (i.e., $K=10^{12}$ in the simulation results).

Another approach for power allocation is related to the possibility of transmitting very short pulses, in the order of hundred femtoseconds. Typically, in $\mathrm{THz}$ band, these pulses are modeled following a Gaussian distribution,

$$
p(t)=\frac{A}{\sqrt{2 \pi \sigma^{2}}} e^{-\frac{(t-\mu)^{2}}{2 \sigma^{2}}},
$$

where $\sigma$ is the standard deviation (in seconds) of the Gaussian pulse, $\mu$ is the mean value of the distribution (in seconds) representing the time instant of the center of the pulse, and $A$ is a normalizing constant useful to adjust the pulse total energy. From (14), the p.s.d. of the $n$-order time derivate of a femtosecond-long pulse is also Gaussian shaped, and so the power level in the $i$-th sub-band has the following expression:

$$
P_{i}=P_{\text {pulse }}{ }^{(n)}(f)=A^{2}(2 \pi f)^{2 n} e^{-(2 \pi \sigma f)^{2}} .
$$

This technique is then defined as Pulse-based power allocation. 


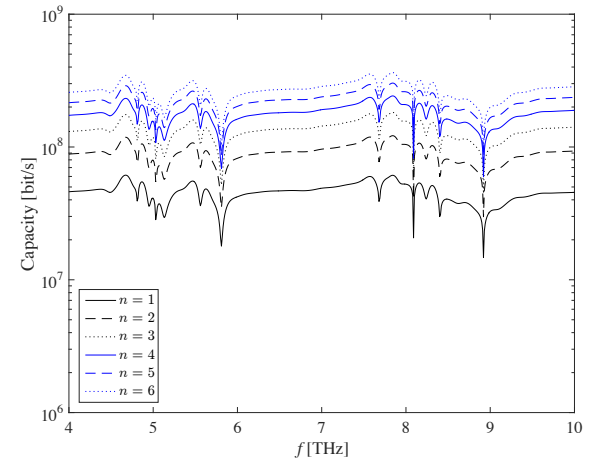

(a)

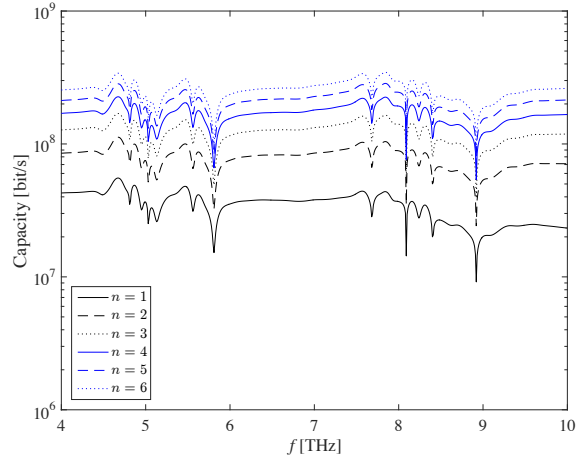

(b)

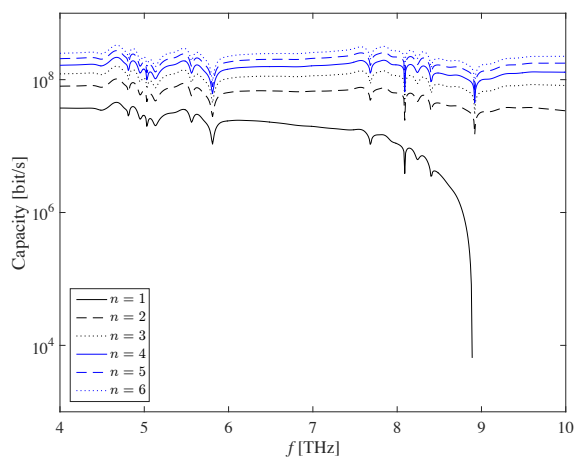

(c)

Fig. 7. Capacity per sub-band in a chirality-affected channel in LoS propagation with GOA, in case of (a) $50 \mathrm{fs}$, (b) $100 \mathrm{fs}$, and (c) $150 \mathrm{fs}$ pulse transmission, for a distance of $1 \mathrm{~mm}$

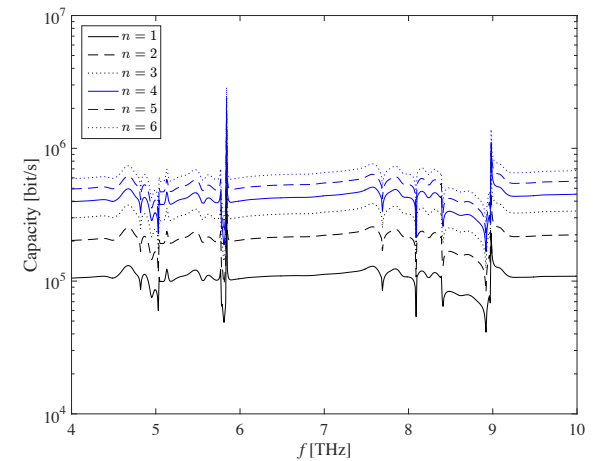

(a)

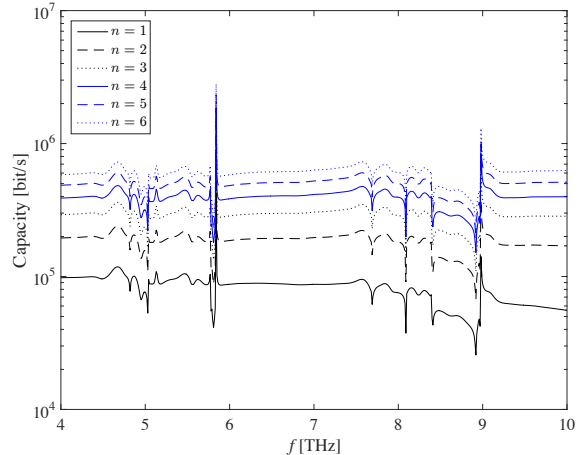

(b)

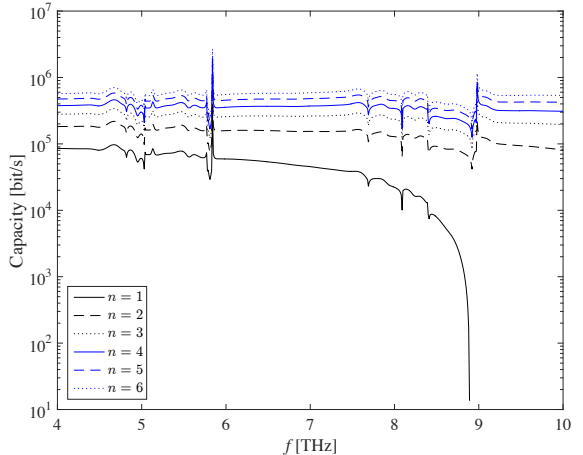

(c)

Fig. 8. Capacity per sub-band in a chirality-affected channel in NLoS propagation with GOA, in case of (a) 50 fs, (b) 100 fs, and (c) 150 fs pulse transmission, for a distance of $1 \mathrm{~mm}$.

As follows, we show the main results of channel capacity per different sub-bands. Table II collects all the relevant parameters used in different simulated scenarios.

Fig. 5 and 6 depict the channel behavior in a chiral affected channel for different p.s.d. schemes, in case of LoS and NLoS propagation with GOA, respectively. Particularly, we consider different time derivative orders for the pulse scheme i.e., $n=1$ and $n=4$, which correspond to a low- and high-order pulse power allocation approach (i.e., LO Pulse and HO Pulse, respectively). A comparison to the case of absence of chirality, i.e., No GOA scenario, is also depicted. As a first result, we notice that the channel capacity in case of No GOA has a flat behavior, slightly increasing for higher frequencies, and decreasing for lower distances. On the other side, due to the frequency-dependent resonant behavior of chiral parameter, the channel capacity in case of chirality-affected channel and GOA shows a variable trend, with peaks due to resonances. However, on average, we observe higher values of channel capacity with respect to the flat behavior obtained with No GOA curves, except for a few peaks below No GOA curves.

The channel capacity per sub-band shows high values in case of $(n=4)$ pulse power allocation scheme and as expected best performances are for lower distances. Particularly, in Fig. 6 (a) we compute the channel capacity per sub-band by assuming a distance of $1 \mathrm{~mm}$ and different p.s.d. schemes.
We observe that best performances are obtained in the case of the pulse p.s.d. for $n=4$. Similar considerations can be taken for other distances, such as in Fig. $6(b)$ and $(c)$ for 5 and $10 \mathrm{~mm}$, respectively, where as expected we notice a decrease of channel capacity for higher distances. The behavior increases in the case of high-order pulse scheme (i.e., $n=4$ ), and low-order pulse transmission (i.e., $n=1$ ), followed by WF, while lowest values are obtained for EP case. We evince that since we can observe that on average the channel capacity per sub-band in the case of GOA outperforms the No GOA scenario, in the next simulations we will focus only on a chirality-affected channel with GOA effect.

In Fig. 7 and 8 we depict the channel capacity for LoS and NLoS propagation, respectively, both in the frequency range $(4-10) \mathrm{THz}$, and assuming different delays of pulses, i.e., 50, 100 and $150 \mathrm{fs}$, and different distances, i.e., 1, 5, and $10 \mathrm{~mm}$. We observe that for lower pulse delays, the channel capacity per sub-band is slightly increased. On the other side, the capacity has a decreasing trend for increasing delays and higher frequencies. This is also noticeable for low derivative number $n$, which causes a strong decrease of capacity specially for highest pulse delays (i.e., $\sigma=150$ fs). Indeed, by computing the $n$-order time derivative of the Gaussian pulse, the corresponding p.s.d. is shifted towards higher frequencies. Also, since the path loss increases with frequency and distance, 


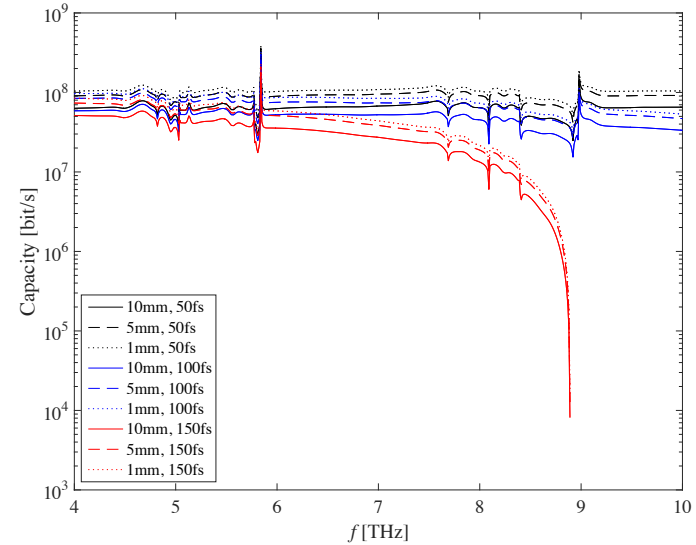

(a)

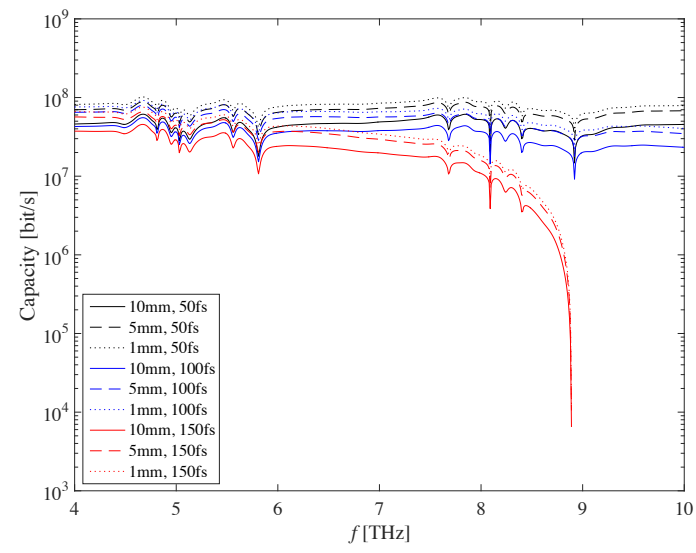

(b)

Fig. 9. Capacity per sub-band in a chiral affected-channel in (a) LoS and (b) NLoS propagation, with GOA for pure pulse transmissions $(n=1)$ and different distances.

the channel capacity per sub-band increases for higher time derivatives. Again, by comparing Fig. 7 and 8, we observe that LoS scenario presents -as expected- higher values of channel capacity per sub-band.

In Fig. 9 (a), we can notice that by increasing the pulse delay the channel capacity shows a decreasing trend for increasing frequencies. Indeed, in LoS the capacity gap in the case of 50 and $100 \mathrm{fs}$ for a distance of $1 \mathrm{~mm}$ is smaller for lower frequencies (e.g., $6.4 \mathrm{Mbit} / \mathrm{s}$ at $4 \mathrm{THz}$ ), while it increases for higher frequencies (e.g., $20.4 \mathrm{Mbit} / \mathrm{s}$ at $6.9 \mathrm{THz}$, and 21.3 Mbit/s at $8.89 \mathrm{THz}$ ). The same consideration is applied in the case of 50 and 150 fs pulses, where we observe that longer delays cause a degradation of channel capacity around 8.89 $\mathrm{THz}$.

A similar consideration can be applied to the case of NLoS propagation as depicted in Fig. 9 (b), where we observe values of capacity similar to those obtained for LoS. Also in this scenario, for longer pulses the capacity experiences lower values, especially for the resonance peak at $8.9 \mathrm{THz}$ where the trend reaches the lowest value $\left(\approx 10^{4} \mathrm{bit} / \mathrm{s}\right)$ for $\sigma=150 \mathrm{fs}$.

Lastly, in Fig. 10 and 11 we show the channel capacity per sub-band in the case of high-order pulse scheme (i.e., $n=$ 4), for LoS and NLoS propagation, respectively. This trend is computed for different time delays and is compared to the low-order pulse scenario (i.e., $n=1$ ) for different distances. We notice that the high-order pulse scheme outperforms the low-order pulse case. When the time delay increases, the trend of channel capacity decreases and a very low performance is reached in the case of $(n=4)$ pulse scheme for long pulse delays. Indeed, for 150 fs pulses and at higher frequencies the capacity behavior is strongly decreased. On the other side, for $(n=1)$ pulse scenario, the performance is still acceptable also for higher frequencies.

To summarize, in Table III we collect the values of the channel capacity sampled at the frequency of $7.9 \mathrm{THz}$, corresponding to one resonant peak of the chiral parameter and also to the maximum value of channel capacity in case of GOA and different power allocation schemes. Then, Table III shows the values of the channel capacity at $7.9 \mathrm{THz}$ in case of GOA/No GOA, LoS/NLoS propagation, different power allocation schemes, and distances.

\section{CONCLUSIONS}

In this paper, we have presented the performance-expressed in terms of capacity bounds- of a chirality-affected channel with GOA, under the hypothesis of LoS and NLoS propagation in the $(4-10) \mathrm{THz}$ band. We considered the effects of the relative chiral parameter through a frequency-dependent behavior with resonant peaks at specific frequencies.

Simulation results have been assessed for different power allocation schemes, propagation modes, and distances. As a result, we were able to identify specific scenarios that guarantee high performances. Specifically, in LoS with the high-order pulse power allocation scheme (i.e., HO-Pulse), the chirality-affected channel with GOA can guarantee a capacity approaching $10^{9} \mathrm{bit} / \mathrm{s}$, also for higher distances. Also, a comparison to a chiral-metamaterial channel without GOA has shown lower performances, thus motivating researchers to keep on investigating into the field of chirality-affected channels with GOA for $\mathrm{THz}$ communications.

\section{REFERENCES}

[1] I. F. Akyildiz and J. M. Jornet, "The Internet of Nano-Things," IEEE Wireless Communication Magazine, vol. 17, no. 6, pp. 58-63, December 2010.

[2] I. F. Akyildiz, J. M. Jornet, and C. Han, "Terahertz Band: Next Frontier for Wireless Communications," Physical Communication (Elsevier) Journal, vol. 12, pp. 16-32, 2014.

[3] R. Piesiewicz, T. Kleine-Ostmann, N. Krumbholz, D. Mittleman, M. Koch, J. Schoebel, and T. Kurner, "Short-range ultra-broadband terahertz communications: Concepts and perspectives," Antennas and Propagation Magazine, IEEE, vol. 49, no. 6, pp. 24-39, Dec 2007.

[4] J. Jornet and I. Akyildiz, "Channel modeling and capacity analysis for electromagnetic wireless nanonetworks in the terahertz band," Wireless Communications, IEEE Transactions on, vol. 10, no. 10, pp. 3211-3221, Oct. 2011.

[5] I. T. Javed and I. H. Naqvi, "Frequency band selection and channel modeling for wnsn applications using simplenano," in 2013 IEEE International Conference on Communications (ICC), June 2013, pp. 5732-5736.

[6] I. Llatser, A. Mestres, S. Abadal, E. Alarcon, H. Lee, and A. CabellosAparicio, "Time- and frequency-domain analysis of molecular absorption in short-range terahertz communications," Antennas and Wireless Propagation Letters, IEEE, vol. 14, pp. 350-353, 2015. 


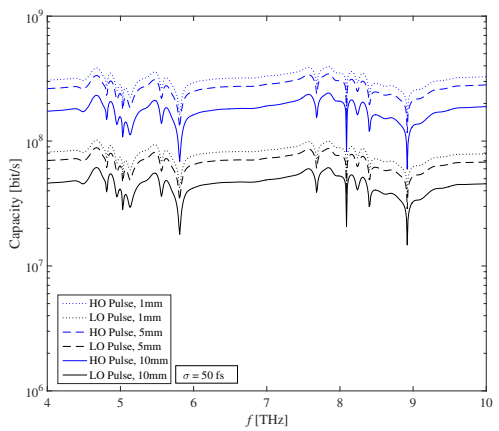

(a)

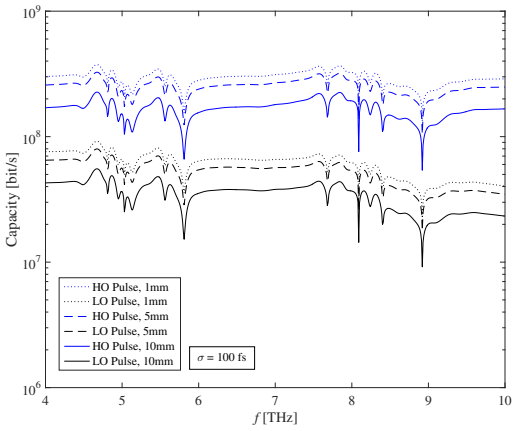

(b)

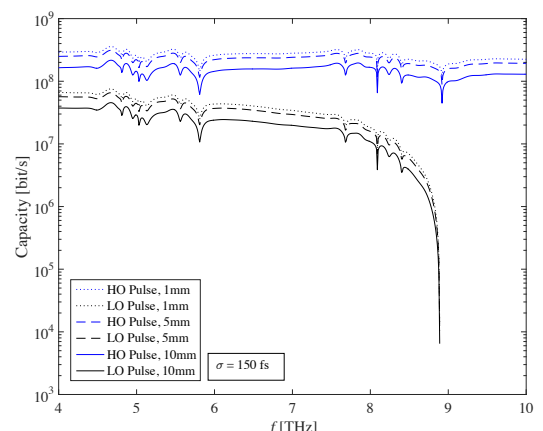

(c)

Fig. 10. Capacity per sub-band in a chirality-affected channel in LoS propagation with GOA, in case of low-order pulse scenario (i.e., $n=1)$ and high-order pulse transmission (i.e., $n=4$ ), for (a) 50, (b) 100, and (c) 150 fs pulses.

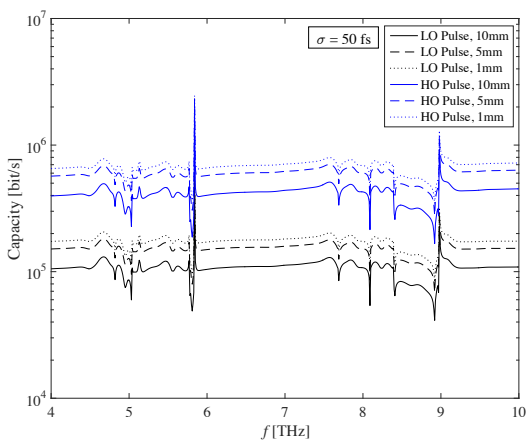

(a)

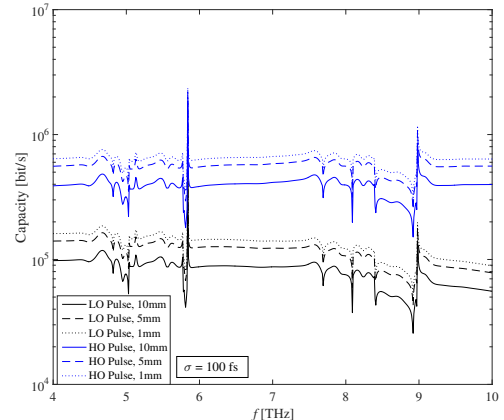

(b)

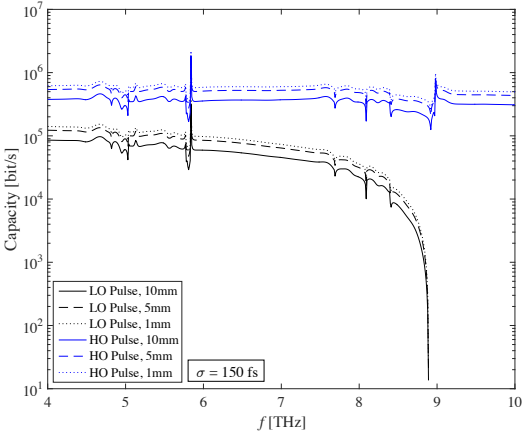

(c)

Fig. 11. Capacity per sub-band in a chirality-affected channel in NLoS propagation with GOA, in case of low-order pulse scenario (i.e., $n=1)$ and high-order pulse transmission (i.e., $n=4$ ), for (a) 50 , (b) 100 , and (c) 150 fs pulses.

TABLE III

CHANNEl CAPACITY VALUES [MBIT/S] SAMPLED AT 7.9 THz, IN CASE OF LOS/NLOS PROPAGATION MODE, GOA/NO GOA, DIFFERENT POWER ALLOCATION SCHEMES, AND DISTANCES.

\begin{tabular}{|c|c|c|c|c|c|c|c|c|}
\hline & Distance & Power allocation & GOA & No GOA & & & GOA & No GOA \\
\hline & & EP & 16.55 & 11.63 & & & 0.0321 & 0.0287 \\
\hline & & WF & 41.94 & 33.85 & & $1 \mathrm{~mm}$ & 0.0928 & 0.083 \\
\hline & $1 \mathrm{~mm}$ & Low-order Pulse & 99.8 & 70.11 & & $1 \mathrm{~mm}$ & 0.194 & 0.173 \\
\hline & & High-order Pulse & 395.3 & 277.7 & & & 0.768 & 0.685 \\
\hline & & EP & 14.45 & 9.86 & & & 0.0283 & 0.0251 \\
\hline LoS & & WF & 36.8 & 28.78 & NLoS & & 0.082 & 0.0727 \\
\hline & $5 \mathrm{~mm}$ & Low-order Pulse & 87.15 & 59.46 & & $5 \mathrm{~mm}$ & 0.171 & 0.151 \\
\hline & & High-order Pulse & 345.2 & 235.5 & & & 0.677 & 0.599 \\
\hline & & $\mathrm{EP}$ & 10.15 & 6.326 & & & 0.0205 & 0.0176 \\
\hline & $10 \mathrm{~mm}$ & WF & 26.19 & 18.6 & & $10 \mathrm{~m}$ & 0.0592 & 0.0515 \\
\hline & $10 \mathrm{~mm}$ & Low-order Pulse & 61.23 & 38.15 & & $10 \mathrm{il}$ & 0.123 & 0.106 \\
\hline & & High-order Pulse & 242.5 & 151.1 & & & 0.489 & 0.421 \\
\hline
\end{tabular}

[7] K. Yang, Q. H. Abbasi, K. Qaraqe, A. Alomainy, and Y. Hao, "Bodycentric nano-networks: Em channel characterisation in water at the terahertz band," in 2014 Asia-Pacific Microwave Conference, Nov 2014, pp. 531-533.

[8] G. Piro, K. Yang, G. Boggia, N. Chopra, L. Grieco, and A. Alomainy, "Terahertz communications in human tissues at the nanoscale for healthcare applications," Nanotechnology, IEEE Transactions on, vol. 14, no. 3, pp. 404-406, May 2015.

[9] I. Llatser, A. Cabellos-Aparicio, E. Alarcon, J. M. Jornet, A. Mestres, H. Lee, and J. Sole-Pareta, "Scalability of the channel capacity in graphene-enabled wireless communications to the nanoscale," IEEE Transactions on Communications, vol. 63, no. 1, pp. 324-333, Jan 2015.

[10] C. Han, A. Bicen, and I. Akyildiz, "Multi-ray channel modeling and wideband characterization for wireless communications in the terahertz band," Wireless Communications, IEEE Transactions on, vol. 14, no. 5, pp. 2402-2412, May 2015.

[11] C. Zhang, C. Han, and I. F. Akyildiz, "Three dimensional end-toend modeling and directivity analysis for graphene-based antennas in the terahertz band," in 2015 IEEE Global Communications Conference (GLOBECOM), Dec 2015, pp. 1-6.

[12] J. Kokkoniemi, J. Lehtomki, and M. Juntti, "A discussion on molecular absorption noise in the terahertz band," Nano Communication Networks, pp. -, 2015. [Online]. Available: http://www.sciencedirect.com/science/ article/pii/S1878778915000472

[13] N. Khalid and O. B. Akan, "Wideband thz communication channel measurements for 5g indoor wireless networks," in 2016 IEEE International Conference on Communications (ICC), May 2016, pp. 1-6.

[14] S. Priebe, M. Jacob, and T. Krner, "Angular and rms delay spread 
modeling in view of thz indoor communication systems," Radio Science, vol. 49, no. 3, pp. 242-251, 2014, 2013RS005292. [Online]. Available: http://dx.doi.org/10.1002/2013RS005292

[15] T. Krner and S. Priebe, "Towards thz communications - status in research, standardization and regulation," Journal of Infrared, Millimeter, and Terahertz Waves, vol. 35, no. 1, pp. 53-62, 2014. [Online]. Available: http://dx.doi.org/10.1007/s10762-013-0014-3

[16] N. Khalid and O. B. Akan, "Experimental throughput analysis of low-thz mimo communication channel in $5 \mathrm{~g}$ wireless networks," IEEE Wireless Communications Letters, vol. 5, no. 6, pp. 616-619, Dec 2016.

[17] A. Moldovan, M. Ruder, I. Akyildiz, and W. Gerstacker, "Los and nlos channel modeling for terahertz wireless communication with scattered rays," in Globecom Workshops (GC Wkshps), 2014, Dec 2014, pp. 388392.

[18] C. Han, A. Bicen, and I. Akyildiz, "Multi-wideband waveform design for distance adaptive wireless communications in the terahertz band," Signal Processing, IEEE Transactions on, vol. 64, no. 4, pp. 910-922, Febr. 2016.

[19] I. Lindell, A. Sihvola, S. Tretyakov, and A. Viitanen, Electromagnetic waves in chiral and bi-isotropic media. Artech House, 1994.

[20] F. Fang and Y. Cheng, "Dual-band terahertz chiral metamaterial with giant optical activity and negative refractive index based on cross-wire structure," Progress In Electromagnetics Research M, vol. 31, pp. 59-69, 2013.

[21] E. Plum, M. Ren, V. Fedotov, J. Xu, and N. Zheludev, "Giant linear and nonlinear optical activity in metamaterials," April 2012. [Online]. Available: http://eprints.soton.ac.uk/379293/

[22] B. Wang, T. Koschny, M. Kafesaki, and C. M. Soukoulis, "Chiral metamaterials: Simulations and experiments," J. Opt. A: Pure Appl. Opt., vol. 11, 2009.

[23] V. G. Veselago, "The electrodynamics of substances with simultaneously negative values of $\varepsilon$ and $\mu$," Sov. Phys. Usp., vol. 10, no. 4, pp. 509-514, 1968.

[24] F. Martn, "Metamaterials for wireless communications, radiofrequency identification, and sensors," ISRN Electronics, vol. 2012, p. 29, 2012.

[25] M. J. Freire, L. Jelinek, R. Marques, and M. Lapine, "On the application of $\mu_{r}=-1$ metamaterial lenses for magnetic resonance imaging," $J$. Magn. Reson., vol. 203, pp. 81-90, 2010.

[26] B. Wang, W. Yerazunis, and K. H. Teo, "Wireless power transfer: Metamaterials and array of coupled resonators," vol. 101, pp. 13591368, Jun. 2013.

[27] N. Lopez, C. Lee, A. Gummalla, and M. Achour, "Compact metamaterial antenna array for long term evolution (lte) handset application," in Proc. IEEE Int. Workshop Antenna Technol., Santa Monica, CA, USA, Mar. 2009.

[28] W. Withayachumnankul and D. Abbott, "Metamaterials in the terahertz regime," vol. 1, pp. 99-118, August 2009.

[29] F. Capolino, Metamaterials Handbook, Applications of Metamaterials. CRC Press, 2009.

[30] H. Guo, Z. Sun, J. Sun, and N. M. Litchinitser, " $\mathrm{M}^{2} \mathrm{i}$ : Channel modeling for metamaterial-enhanced magnetic induction communications," IEEE Transactions on Antennas and Propagation, vol. 63, no. 11, pp. 5072 5087, Nov 2015.

[31] "Study and fabrication of metamaterials for applications to electronics and telecommunications," National Italian Research Project: PRIN, 2006.

[32] A. Tuniz, R. Lwin, A. Argyros, S. Fleming, and B. Kuhlmey, "Fabricating Metamaterials Using the Fiber Drawing Method," J. Vis. Exp., vol. 68, 2012.

[33] A. M. et al., "Composite thz materials using aligned metallic and semiconductor microwires, experiments and interpretation," Opt Express., vol. 18,2010

[34] G. Kenanakis, R. Zhao, A. Stavrinidis, G. Konstantinidis, N. Katsarakis, M. Kafesaki, C. M. Soukoulis, and E. N. Economou, "Flexible chiral metamaterials in the terahertz regime: a comparative study of various designs," Opt. Mater. Express, vol. 2, no. 12, pp. 1702-1712, Dec 2012. [Online]. Available: http://www.osapublishing.org/ome/abstract. cfm?URI=ome-2-12-1702

[35] B. Wang, J. Zhou, T. Koschny, M. Kafesaki, and C. M. Soukoulis, "Chiral metamaterials: simulations and experiments," J. Opt. A: Pure Appl. Opt., vol. 11, p. 10, 2009.

[36] Z. Wang, F. Cheng, T. Winsor, and Y. Liu, "Optical chiral metamaterials: a review of the fundamentals, fabrication methods and applications," Nanotechnology, vol. 27, p. 20, 2016.

[37] Z. Li, R. Zhao, T. Koschny, M. Kafesaki, K. B. Alici, E. Colak, H. Caglayan, E. Ozbay, and C. M. Soukoulis), "Chiral metamaterials with negative refractive index based on four "U" split ring resonators," Appl. Phys. Lett., vol. 97, 2010.
[38] J. Watson and F. Crick, "A structure for deoxyribose nucleic acid," Nature, vol. 171, no. 9, pp. 737-738, 1953.

[39] A. M. Vegni and V. Loscrí, "Chirality effects on channel modeling for thz-band wireless communications in los/nlos propagation," Nano Communication Networks, vol. 10, pp. 27 - 37, 2016.

[40] —, "Performance of a Chirality-affected Channel exhibiting Giant Optical Activity for Terahertz Communications," in Proc. of ACM NanoCom 2016, Sept. 2016, pp. 1-6.

[41] A. M. Vegni and V. Loscr, "Characterization and performance analysis of a chiral-metamaterial channel with giant optical activity for terahertz communications," Nano Communication Networks, vol. 9, pp. 28 - 35, 2016. [Online]. Available: //www.sciencedirect.com/science/article/pii/ S1878778916300217

[42] J. Zhou, D. R. Chowdhury, R. Zhao, A. Azad, H.-T. Chen, C. M. Soukoulis, and A. J. Taylor, "Terahertz chiral metamaterials with giant and dynamically tunable optical activity," Phys. Rev. B, vol. 86, Jul 2012. [Online]. Available: http://link.aps.org/doi/10.1103/PhysRevB.86. 035448

[43] R. Zhao, T. Koschny, and C. M. Soukoulis, "Chiral metamaterials: retrieval of the effective parameters with and without substrate," Optics express, vol. 18, no. 4, July 2010.

[44] J. Margineda, G. Molina-Cuberos, M. Nez, A. Garca-Collado, and E. Martn, "Electromagnetic characterization of chiral media," in Solutions and Applications of Scattering, Propagation, Radiation and Emission of Electromagnetic Waves, A. Kishk, Ed. InTech, 2012, ch. 1.

[45] P. Drude, Lehrbuch der Optik, 1900.

[46] B.-J. Lee, J.-P. Cho, I.-H. Ra, and K.-S. Kim, "Propagation Characterization Based on Geographic Location Variation for 5G Small Cells," Mobile Information Systems, vol. 2017, p. 8, 2017.

[47] F. Fang, Y. Cheng, and H. Liao, "Giant optical activity and circular dichroism in the terahertz region based on bi-layer Y-shaped chiral metamaterial," Optik-Int. J. Light Electron Opt., 2014.

[48] Y. Z. Cheng, Y. L. Yang, Y. J. Zhou, Z. Zhang, X. S. Mao, and R. Z. Gong, "Complementary Y-shaped chiral metamaterial with giant optical activity and circular dichroism simultaneously for terahertz waves," Journal of Modern Optics, 2016.

[49] Y. Z. Cheng, Y. Nie, and R. Z. Gong, "Giant optical activity and negative refractive index using complementary U-shaped structure assembly," Progress In Electromagnetics Research M, vol. 25, pp. 239-253, 2012.

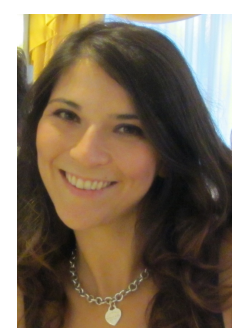

Anna Maria Vegni is non-tenured Assistant Professor in Telecommunications at the Dept. of Engineering of Roma Tre University, Italy, where she received the Ph.D. degree in Biomedical Engineering, Electromagnetic and Telecommunications in 2010 In 2009 , she was a visiting researcher at the Dept. of Electrical and Computer Engineering, Boston University (USA), under the supervision of Prof. T. Little. She is involved in several EU projects, and organization committees of international conferences. Her research activity focuses on wireless networks, Visible Light Communications, and nanocommunications. She is in the editorial board of Elsevier Ad Hoc Networks, JNCA and NANOCOMNET.

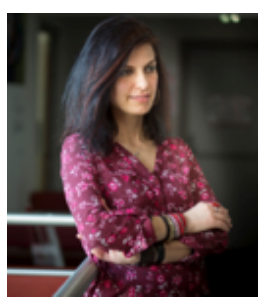

Valeria Loscrí is a permanent researcher of the FUN Team at Inria Lille-Nord Europe since Oct. 2013. From Dec. 2006 to Sept. 2013, she was Fellow in the TITAN Lab of the University of Calabria, Italy. She received her MSc and $\mathrm{PhD}$ degrees in Computer Science in 2003 and 2007, respectively, from the University of Calabria. Her research interests focus on heterogeneous communication technologies and cooperation of heterogeneous devices. She has been involved in the FP7 EU project VITAL, the FP6 EU project MASCOT, the national project (PRIN) Stem-

NET and (PON) Kom4t-me. She is in the editorial board of Elsevier ComNet, JNCA, IEEE Trans. on Nanobioscience. Since 2016, she is Scientific European Responsible for Inria Lille-Nord Europe. 